LBL- -29650

DE9 I 007748

\title{
Research Opportunities at the Advanced Light Source*
}

Arthur L. Fobinson and Alfred S. Schlachter

\author{
Advanced Light Source \\ Accelerator and Fusion Research Division \\ Lawrence Berkeley Laboratory \\ 1 Cyclotron Road \\ Berkeley, CA 94720
}

September 26, 1990

Paper to be Presented at the 11th International Conference on the Application of Accelerators in Research and Industry, Denton, Texas, November 5-8, 1990
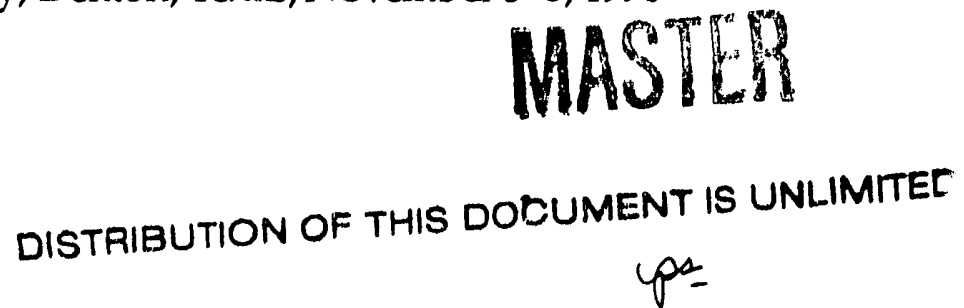

"This work was supported by the Director, Office of Energy Research, Office of Basic Energy Sciences, Materials Sciences Division of the U.S. Department of Energy, under Contract No. DE-AC03-76SF00098 


\section{RESEARCH OPPORTUNITLES AT THE ADVANCED LIGHT SOURCE*}

\section{A.L. ROBINSON AND A.S. SCHLACHTER}

Advanced Light Source, Accelerator and Fusion Research Division, Lawrence Berkeley Laboratory, University of California, Berkeley, CA 94720 USA

The Advanced Light Source (ALS), now under construction at the Lawrence Berkeley Laboratory, is a third-generation synchrotron radiation facility based on a low-emittance, $1.5-\mathrm{GeV}$ electron storage ring with 10 long straight sections available for insertion devices and, initially, 24 bendmagnet ports. Undulators will provide high-brightness radiation at photon energies from below $10 \mathrm{eV}$ to above $2 \mathrm{keV}$; wiggler and bend-magnet radiation will extend the spectral coverage with high fluxes to above $10 \mathrm{keV}$. Scheduled to begin operations as a U.S. Department of Energy national user facility in the spring of 1993 , the ALS will support an extensive research program in which soft $\mathrm{x}$-ray and ultraviolet radiation is used to study matter in all its varied gaseous, liquid, and solid forms. Participating research teams to implement the initial scientific program have been selected.

* This work was supported by the Director, Office of Energy Research, Office of Basic Energy Sciences, Division of Materials Sciences, of the U.S. Department of Energy under Contract No. DE-AC03-76SF00098. 


\section{Introduction}

Around the world, construction of several third-generation synchrotronradiation sources is either under way or planned. These facilities are designed to maximize the spectral brightness (flux per unit area of the source, per unit solid angle of the radiation cone, and per unit bandwidth) of $\mathrm{x}$-ray and ultraviolet radiation by means of an electron or positron storage ring specifically designed to have very low emittance and several long straight sections primarily for undulators. This combination can generate radiation with a spectral brightness that is a factor of 20 or more greater than that of existing, second-generation sources, depending on the spectral range [1]. In the past, order-of-magnitude increases in brightness have led to qualitatively new developments in spectroscopic and structural studies of both gas-phase and condensed matter [2].

The Advanced Light Source (ALS) at the Lawrence Berkeley Laboratory will be a third-generation synchrotron facility optimized for the production of soft $\mathrm{x}$-rays and ultraviolet radiation. The ALS is intended to be a national user facility upen to all qualified scientists and technologists when operations begin in the spring of 1993. The facility consists of injector accelerators, a $1.5 \mathrm{GeV}$ electron storage ring, a complement of undulators and wigglers (collectively, insertion devices), beamlines, and associated experimental apparatus, and a building to house this equipment. The ALS is described in detail in a conceptual design report [3].

\section{Scientific program}

The initial scientific program at the ALS is being implement by means of participating research teams (PRTs) consisting of investigators with 
related research interests from one or more institutions. The primary responsibility for experimental apparatus (i.e., apparatus downstream from the exit slit of the monochromator) rests with the PRTs; the responsibility for the beamlines and insertion devices will be shared between the ALS and the PRTs. Each PRT will be assigned, for its own research program, privileged access to the facilities it helps develop. The amount of access will depend on the resources (personnel, funding, and equipment) provided for the facilities by the PRT. However, a substantial fraction of the beam time at every beamline will be allocated to independent investigators by means of a proposal-review process.

Proposals were invited from candidate PRTs in March 1989. Nine initial insertion-device teams were selected in December 1989, which subsequently coalesced into the eight that are listed in Table 1. As of June 1990, seven bend-magnet ports have also been allocated to PRTs.

Some of these groups are expected to use the increased brightness of the ALS undulators to open new areas of research in the materials sciences, such as spatially resolved photon and electron spectroscopy (spectromicroscopy) [4]. Biological applications will include $x$-ray microscopy with element-specific sensitivity in the water window of the spectrum (23-44 $\AA$ ) where water is much more transparent than protein [5, 6]. The ALS will also be an excellent research tool for atomic physics and chemistry because the high flux will allow measurements to be made with tenuous gas-phase targets $[7,8]$. The short pulse width (30-50 ps) will facilitate time-resolved experiments $[8,9]$.

Other research areas planned for ALS undulator beamlines include high-resolution soft $\mathrm{x}$-ray spectroscopy of materials and surfaces, spin- 
polarized photoemission spectroscopy of magnetic materials, and

experiments that exploit the polarization of undulator radiation. A future option is the construction of special devices to generate radiation with a controlled elliptical polarization. Wiggler-based $\mathrm{x}$-ray studies will include spectroscopy of atoms in both the gas phase and in condensed matter, spatially-resolved elemental analysis with an $\mathrm{x}$-ray microprobe, grazingincidence $\mathrm{x}$-ray scattering from surfaces, and $\mathrm{x}$-ray diffraction of large biological molecules (protein crystallography). Bend-magnet research will include studies of physical and biological systems with polarized radiation and infrared absorption spectroscopy of solids, surfaces and gases.

\section{Photon sources}

The ALS lattice is optimized for the use of insertion devices. Of the 12 straight sections in the storage ring, one is used for injection and one is occupied by rf cavities, leavin 10 full straight sections available for undulators and wigglers up to $4.5 \mathrm{~m}$ in length. Operating at $1.5 \mathrm{GeV}$, the ALS is optimized for insertion-device operation in the soft $\mathrm{x}$-ray and ultraviolet spectral regions.

The spectrum of undulator radiation consists of a series of narrow peaks, a fundamental and its harmonics. The spectrum at an observation angle $\theta$ is given by

$\varepsilon_{n}[\mathrm{keV}]=\frac{0.949 n E^{2}[\mathrm{GeV}]}{\lambda_{u}[\mathrm{~cm}]}\left(\frac{1}{1+K^{2} / 2+\gamma^{2} \theta^{2}}\right)$

where $\varepsilon_{n}$ is the photon energy of the $n$th harmonic, $E$ is the electron-beam 
energy, $K$ is the deflection parameter, which is proportional to the undulator magnetic field and is usually about equal to $1, \lambda_{u}$ is the period of the undulator, and $\gamma$ is the ratio of the beam energy to the energy of the electron rest mass. The relative bandwidth $\Delta \varepsilon / \varepsilon$ of each peak is approximately

$\Delta \varepsilon / \varepsilon=0.9 / n N$ where $N$ is the number of periods.

The spectral range of the undulator is scanned by varying the undulator magnetic field, which decreases as the gap between the poles of the undulator increases. Scanning from low to high photon energies is therefore accomplished by moving the gap from a minimum to a maximum distance. At the ALS, use of the third and fifth harmonics of the undulators is planned to extend their spectral range to higher photon energies than can be reached with the fundamental alone. The wiggler, which operates with a high magnetic field and hence a $\mathrm{K}$ value much greater than 1, generates a broad continuous spectrum characterized by a critical photon energy $\varepsilon_{c}$, defined as the photon energy above which half the total power is radiated. At the high end of the broad wiggler spectrum, the flux drops rapidly but is still one-tenth of its maximum value at photon energies near $4 \varepsilon_{c}$.

Based on the user requirements expressed in proposals received from participating research teams, engineering designs are well under way for insertion devices that cover the ALS spectral range (fig. 1). The goals of very high brightness and useful fifth-harmonic output impose unusually 
tight tolerances on the magnetic-field quality and thus on the mechanical structure of the undulators $[10,11]$. Table 2 lists the properties of three undulators (U8.0, U5.0, and U8.0, where the number refers to the period length in centimeters) that span the soft $\mathrm{x}$-ray and ultraviolet spectral regions when the ALS operates at $1.5 \mathrm{GeV}$. In addition, undulator $\mathrm{U} 10.0$, also included in table 2, has been requested by the chemical dynamics PRT listed in table 1 to provide low-energy photons in the range 6-25 eV. The wiggler in table 2 , with an $\varepsilon_{c}$ of $3.1 \mathrm{keV}$, extends spectral coverage into the hard $\mathrm{x}$-ray region beyond $10 \mathrm{keV}$. High-quality synchrotron radiation will be available from the 24 bend-magnet ports, as well. The critical photon energy of the bend magnets is $1.56 \mathrm{keV}$.

Spectral brightness is shown in fig. 2 for the ALS undulators and wiggler described in table 2, the ALS bending magnets, and for representative sources at other facilities. The spectral brightness of the ALS undulators is shown in more detail in fig. 3 , which separately shows the fundamental and the third and fifth harmonics.

\section{Insertion-device beam lines}

Low-emittance storage rings and insertion devices have created new challenges for designers of UV and soft X-ray optics. The source size and divergence have become smaller. For ALS undulators at the high-photonenergy end cf the spectral range, the size is typically $330 \mu \mathrm{m}$ horizontal by $65 \mu \mathrm{m}$ vertical and the divergence is typically $40 \mu \mathrm{rad}$ horizontal by $30 \mu \mathrm{rad}$ vertical $[12,13]$. The small source size requires tighter tolerances for relay optics and monochromator components in both optical figure and finish to avoid loss of light (e.g., rms surface roughness $\approx 0.5 \mathrm{~nm}$ and tangential 
slope error $<1 \mu \mathrm{rad}$ for a condensing mirror). The attainment of higher resolution by use of smaller slits also becomes practical (the spectralresolution gual of monochromators in undulator beamlines is $\Delta \mathrm{E} / \mathrm{E}=10^{-4}$ ). Monochromator components therefore need tighter tolerances to avoid loss of resolution. Finally, the photon-beam power has increased to several $\mathrm{kw} / \mathrm{cm}^{2}$. The requirement that thermal distortions and stress be controlled complicates the design [for example, water cooling in a UHV ( $\approx 1 \mathrm{ntorr}$ ) environment] and limits the choice of materials.

The most serious limitation is that of optical fabrication tolerances. It is difficult to fabricate aspheric optical surfaces (paraboloids, ellipsoids, toroids, etc.) sufficiently accurately in mirrors, monochromators, and other optical elements to take full advantage of the undulator source. One way to address this problem is to avoid the use of aspheric surfaces and to build beamlines entirely with plane and spherical surfaces. Although studies at LBL and elsewhere have confirmed that, when a large heat load is present, thermal considerations dominate the design and cost of the beamline, undulators usually generate lower total power than wigglers. For the ALS undulator beamlines, the power problem is significant but within the state of the art.

To meet these challenges, the initial ALS-constructed undulator beamlines are based on the spherical-grating monochromator system with water-cooling. The strategy is to use spherical surfaces for all mirrors and gratings and to actively water cool the optics. Depending on the beamline requirements, the condensing system chosen for ALS undulator beamlines consists of one or two spherical mirrors. (PRTs are free to choose other optical designs for their beamlines, subject to approval by the ALS.) 
Because of the low emittance of the ALS storage ring, the monochromator can accept the entire undulator beam in most cases, even a a slit-width of $10 \mu \mathrm{m}$.

\section{For more information}

Proposals to form both insertion-device and bend-magnet PRTs continue to le accepted. For information on forming a new PRT or joining an existing one, contact the authors. Information about independentinvestigator proposals will be available at a later date.

This work was supported by the Director, Office of Energy Research, Office of Basic Energy Sciences, Division of Materials Sciences, of the U.S. Department of Energy under Contract No. DE-AC03-76SF00098.

\section{References}

[1] An ALS Handbook, PUB-643 Rev. 2 (Lawrence Berkeley Laboratory, Berkeley, CA 1989).

[2]. 1-2 GeV Synchrotron Radiation Source, PUB-5172 Rev. (Lawrence Berkeley Laboratory, Berkeley, CA, 1986). Note that the values of some ALS parameters have changed since the issuance of this report. See also "The Advanced Light Source at the Lawrence Berkeley Laboratory" at this conference by A. Jackson, pp xx-xx.

[3] H. Winick, Sci. Am. November 1987, pp. 88-99.

[4] G. Margaritondo and F. Cerrina, Nucl. Instr. Meth. A291 (1990) 26.

[5] X-ray Imaging for the Life Science, eds. D. Attwood and B. Barton, LBL-27660 (Lawrence Berkeley Laboratory, Berkeley, CA, 1989). 
[6] The ALS Life Science Center: Conceptual Design Report, PUB-5234 (Lawrence Berkeley Laboratory, 1989).

[7] A.S. Schlachter and A.L. Robinson, Nucl. Instr. Meth. B43 (1989) 450.

[8] Combustion Dynamics Facility: Scientific Program Summary, PUB5284 (Lawrence Berkeley Laboratory, Berkeley, CA, 1990).

[9] V. Rehn, R.A. Rosenberg, and R.S. Williams, Nucl. Instr. Meth. A291 (1990) 50.

[10] U5.0 Undulator Conceptual Design Report, PUB-5256 (Lawrence Berkeley Laboratory, Berkeley, CA 94720, 1989).

[11] U8.0 Undulator Conceptual Design Report, PUB-5276 (Lawrence Berkeley Laboratory, Berkeley, CA 94720, 1990).

[12] T.Warwick, R. DiGennaro, and M. Howells, U5 Beamline Design Document, LBL-040A (Advanced Light Source, Lawrence Berkeley Laboratory, Berkeley, CA, 1989).

[13] T.Warwick, R. DiGennaro, and M. Howells, U5 Beamline Design Document, LSBL-041 (Advanced Light Source, Lawrence Berkeley Laboratory, Berkeley, CA, 1989). 
Table 1

ALS insertion device teams

Name Scientific Focus Spokesperson

U10.0 Chemical dynamics

U8.0 Atoms, molecules, ions

U8.0 Pump-probe, timing, dynamics experiments

U5.0 Surfaces and interfaces

U5.0 Surfaces and interfaces

U3.9 X-ray imaging and optics for life and physical sciences

W16 Atomic, molecular, optical physics; materials science

W16 Life sciences
Tomas Baer, U. of North Carolina

Denise Caldwell, U. of Central Florida

Victor Rehn, Naval Weapons Center

Brian Tonner, U. of Wisconsin-Milwaukee

Joachim Stöhr, IBM Almaden Research Center

Stephen Rothman, U. of California, San Francisco

Bernd Crasemann, U. of Oregon; Philip Ross, LBL

Stephen Cramer, Brookhaven National La'o 
A. L. Robinson and A. S. Schlachter

Table 2

Parameters for initial ALS insertion devices

\begin{tabular}{|c|c|c|c|c|}
\hline Name & $\begin{array}{l}\text { Period } \\
{[\mathrm{cm}]}\end{array}$ & $\begin{array}{l}\text { No. of } \\
\text { periods }\end{array}$ & $\begin{array}{l}\text { Photon energy } \\
\text { range }[\mathrm{eV}] \text { a) }\end{array}$ & $\begin{array}{l}\text { Critical } \\
\text { energy }[\mathrm{keV}]\end{array}$ \\
\hline \multicolumn{5}{|c|}{ Undulators } \\
\hline U10.0 & 10.0 & 45 & -b) & - \\
\hline U8.0 & 8.0 & 55 & $\begin{array}{l}5.4-220 \mathrm{c}) \\
{[16.2-660]} \\
{[27-1100]}\end{array}$ & - \\
\hline U5.0 & 5.0 & 89 & $\begin{array}{c}52-380 \\
{[155-1140]} \\
{[260-1900]}\end{array}$ & - \\
\hline U3.9 & 3.9 & 115 & $\begin{array}{c}169-500 \\
{[507-1500]} \\
{[845-2500]}\end{array}$ & - \\
\hline
\end{tabular}

Wiggler

W16

16

16

$-$

3.1

a) The photon energy range of the fundamental and of the third and fifth harmonics (shown in brackets) as the deflection parameter $\mathrm{K}$ decreases from its maximum value to approximately 0.5 , when the electron-beam energy is $1.5 \mathrm{GeV}$.

b) Only the range from 6 to $25 \mathrm{eV}$ in the fundamental will be used.

c) Below about $8 \mathrm{eV}$ in the fundamental, the peak field in undulator U8.0 exceeds the bending-magnet field and may adversely affect storage-ring operation. 


\section{[FIGURE CAPTIONS]}

Fig. 1. Drawing of a generic insertion device for the straight sections of the ALS storage ring showing the main structural features that ALSconstructed undulators and wigglers will have in common.

Fig. 2. Spectral brightness as a function of photon energy for the three ALS undulators and wiggler described in table 2, the ALS bending magnets, undulators plarned for the Advanced Photon Source (APS), and rnpresentative insertion-device and bend-magnet sources at the National Synchrotron Light Source (NSLS) and the Stanford Syrichrotron Radiation Center (SSRL). X-1 is a recently installed undulator beamline at NSLS.

Fig. 3. Spectral urightness as a function of photon energy for the three undulators described in table 2. Each undulator curve is the locus of narrow peaks of radiation, tuned by altering the undulator gap. Separate curves are shows for the fundamental and the third and fifth harmonics of each undulatnr. The dotted extension of the fundamental of U8.0 represents the performance when the undulator field exceeds the bendmagnet field, a condition that may adversely affect storage-ring operation. 


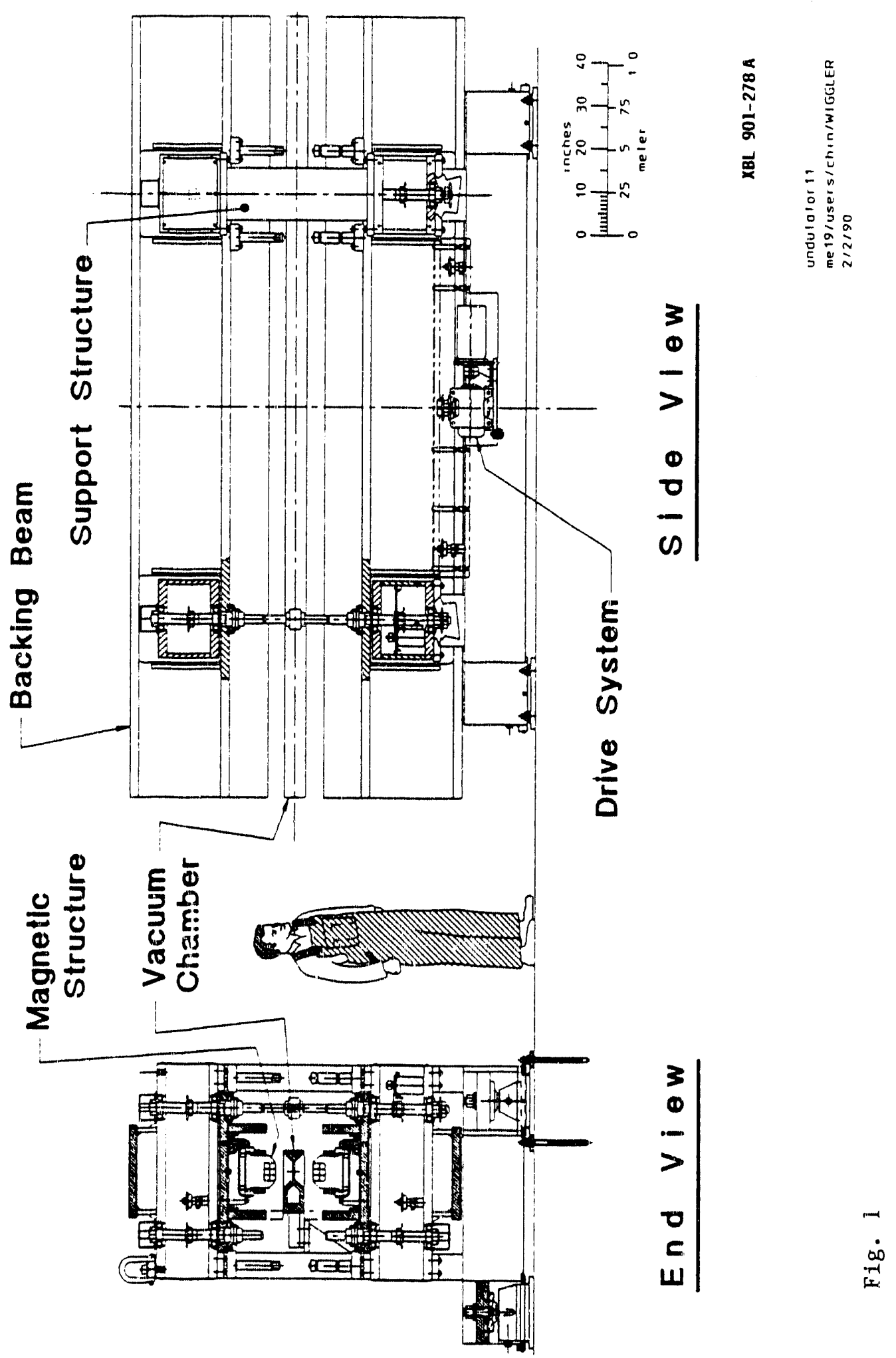




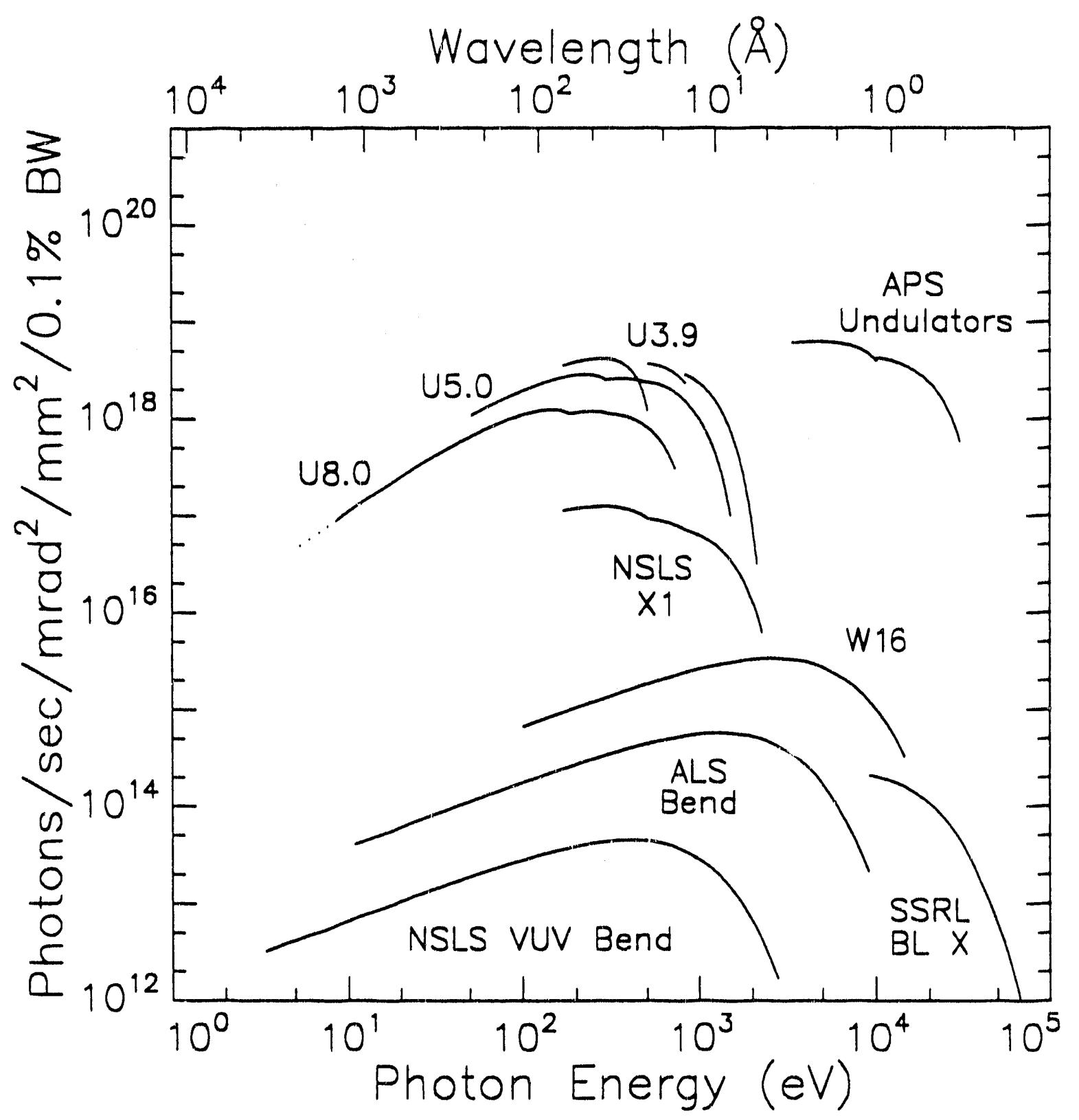

Fig. 2 


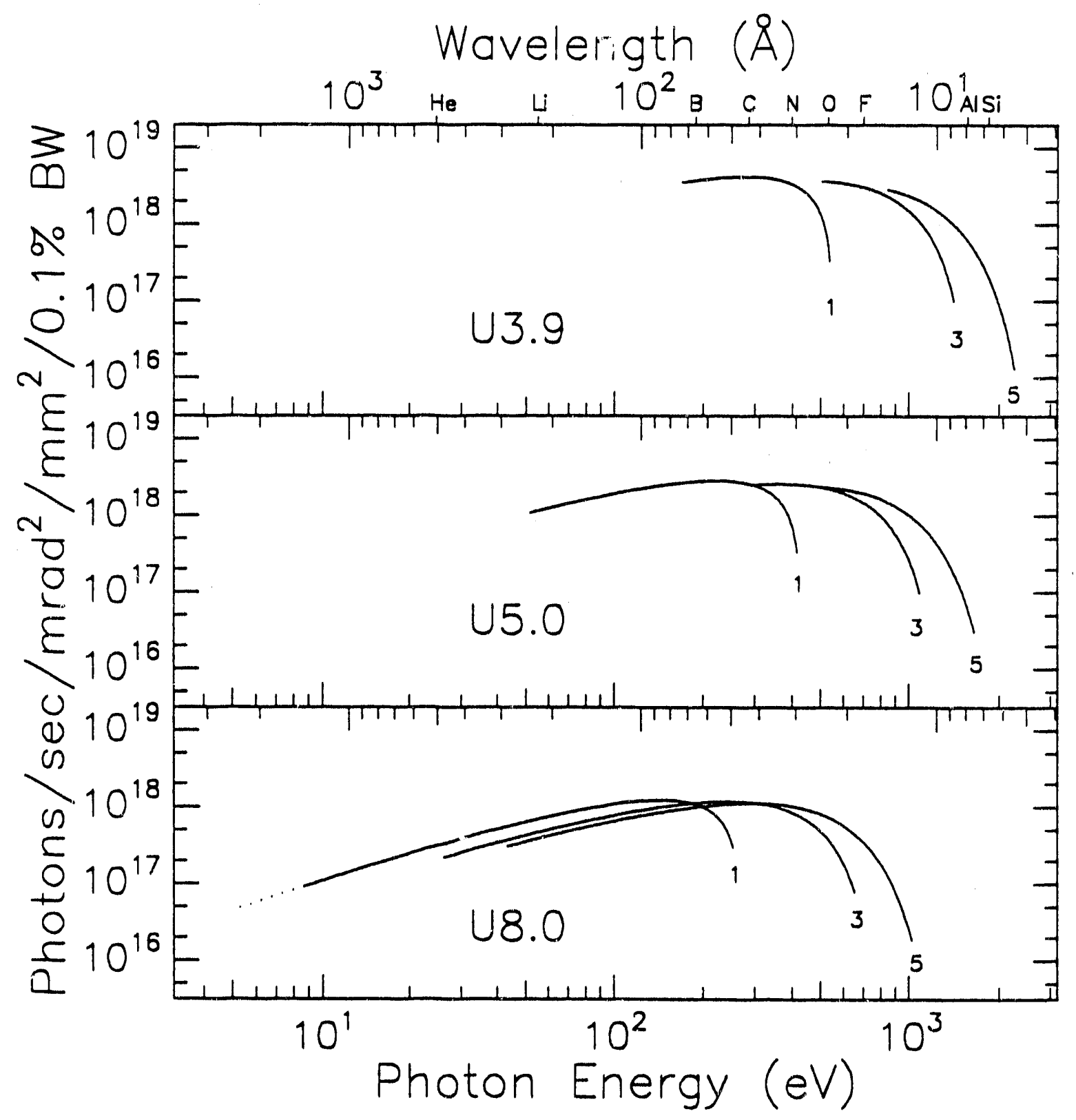

Fig. 3

XBL 893-7096 

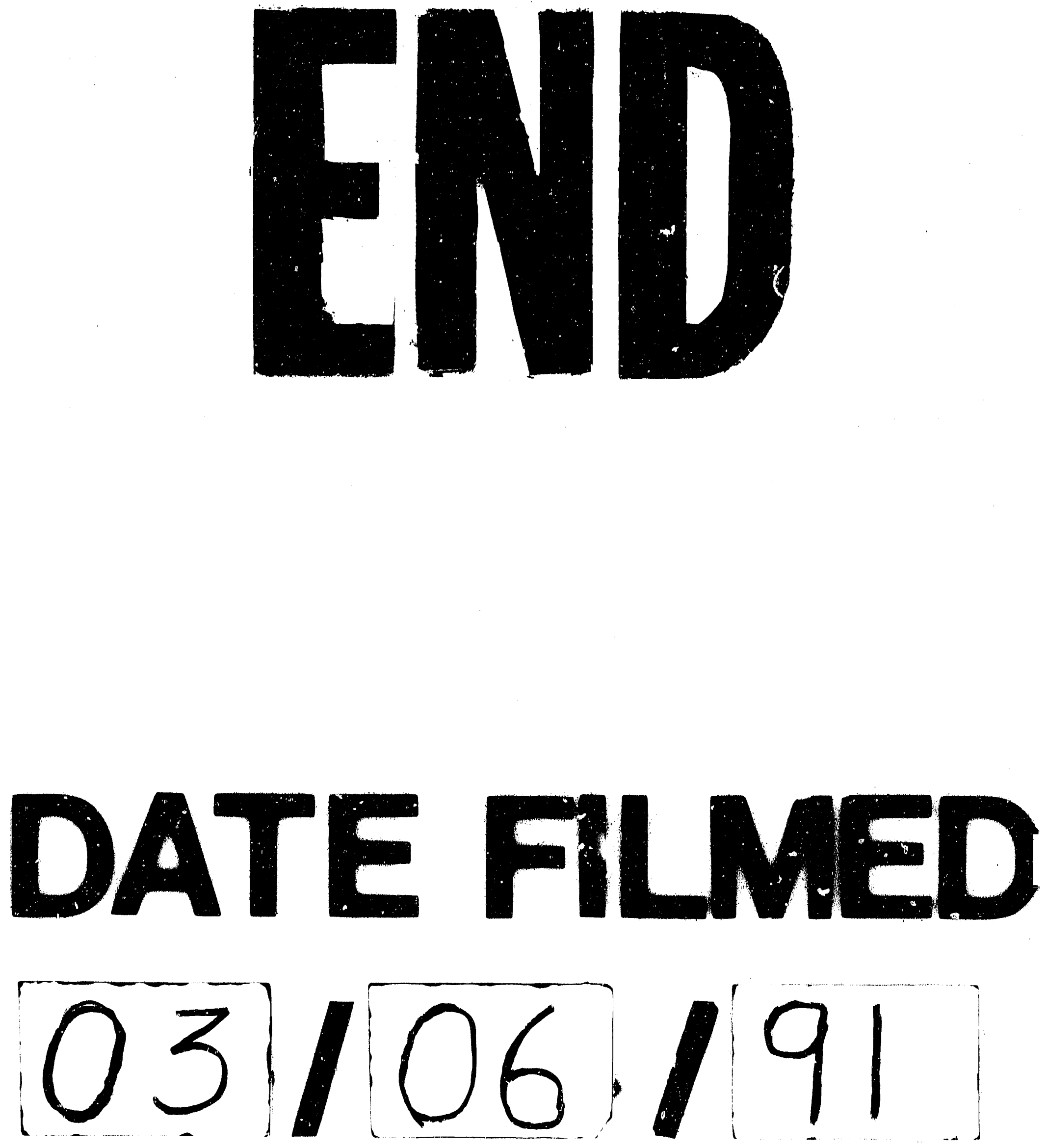
